\title{
Spatial Analysis of Data on the Basis of the Digital Model of Relief and Locality (Example of Shemakha, Akhsu and Ismayilli Districts)
}

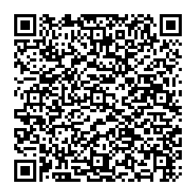

\section{Dr ZH Aliyev*}

Institute of Soil Science and Agrochemistry of NAS of Azerbaijan, Azerbaijan

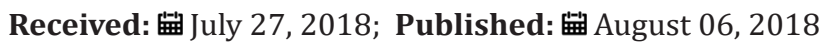

*Corresponding author: Dr ZH Aliyev Institute of Soil Science and Agrochemistry of NAS of Azerbaijan, Azerbaijan

\section{Abstract}

The article examines the issues of studying the degree of susceptibility of sloping lands in Azerbaijan in the example of specific administrative territorial units, flat areas with a slope of up to 6\% (about 3.50) are concentrated in Akhsu district 71\%, and in Shemakha almost $49 \%$. The steepest slopes are observed in the Ismaili region, where almost $26 \%$ of the territory has a slope of $10-18 \%, 30 \%$ of its area slope is $18 \%$.

Keywords: Sloping slopes; Arable lands; A layer; A database; A Gradient class; Soil; Forest pastures; Geese; Cartographic materials

\section{Introduction}

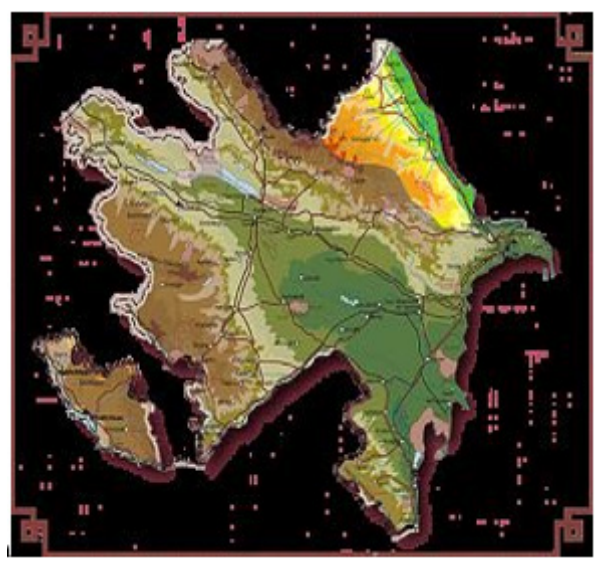

Figure 1

Figure 1 Spatial analysis includes operations performed on geographic data using available methods and techniques in GIS software, with a view to describing the relationships between elements of the geographical environment. The analysis can be carried out on the data, both in the vector and raster systems, and touch the geometry and attributes of the vector data [1-8].

\section{Search for Information in the Database}

The main operation that can be performed based on the GIS database is information retrieval. This database has a relational nature and therefore, the object designation provokes the selection of the corresponding records from the corresponding attribute table, and vice versa. The first thematic layer in the database was the Digital Elevation Model in raster format. On its basis, as a result of the transformations and calculations, information on the height of the nos. and the slope of the terrain on the territory of Azerbaijan selected for analysis, which includes three regions: Shemakha, Akhsu and Ismayilli.

\section{Vertical Position Factor}

The major part of the study area is in the following ranges of heights: 1) from 0 to 300 mas-23.16\%, from 300 to $600 \mathrm{~m}-19.84 \%$ and 3) $600-900 \mathrm{~m}-23,75 \%$ of the total area. Above $900 \mathrm{~m}$ and up to $3400 \mathrm{~m}$ above sea level, about $30 \%$ of its area is located (Table 1). Of the administrative units belonging to the study area, the most highly allocated land Ismaili region. In this area 90: it is at an altitude of more than 300 meters above sea level, and sometimes the altitude is even higher than $3400 \mathrm{~m}$. In Akhsu region about 73\% of the earth is at an altitude up to the sea level. Shemakha district is an intermediate region, its largest area is at an altitude of 900 $\mathrm{m}$ above sea level-about $75 \%$. In this area there is also a territory located below sea level-31244.66 hectares [8-18].

\section{Classes of Slope}

In the analyzed territory, the largest area is occupied by areas with a slope of up to 6-46\%. Significant areas are also in the intervals from 6 to $10 \%-18 \%$ and from $18 \%$ to almost $23 \%$ of the total area 
(Table 2). Against the backdrop of administrative-territorial units, gently sloping areas with a slope of up to 6\% (about 3.50) are concentrated in the Akhsu district 71\%, and in Shemakha almost
$49 \%$. The steepest slopes are observed in the Ismaili region, where almost $26 \%$ of the territory has a slope of $10-18 \%, 30 \%$ of its area slope is $18 \%$.

Table 1: The distribution of the study area (ha and \%) over the altitude intervals.

\begin{tabular}{|c|c|c|c|c|c|}
\hline \multirow{2}{*}{\multicolumn{2}{|c|}{ Height, m. н.y.м. }} & \multicolumn{3}{|c|}{ Regions } & \multirow{3}{*}{$\begin{array}{c}\text { Together } \\
31245\end{array}$} \\
\hline & & \multirow{2}{*}{$\begin{array}{c}\text { Shemakha } \\
31245\end{array}$} & \multirow{2}{*}{$\begin{array}{c}\text { Akhsuinsky } \\
0\end{array}$} & \multirow{2}{*}{$\begin{array}{c}\text { Ismailinskiy } \\
0\end{array}$} & \\
\hline & ha & & & & \\
\hline & $\%$ & 8,12 & 0,00 & 0,00 & 5 \\
\hline \multirow{2}{*}{$0-300$} & ha & 69737 & 73029 & 17334 & 160101 \\
\hline & $\%$ & 18,13 & 72,99 & 8,39 & 23 \\
\hline \multirow{2}{*}{$300-600$} & ha & 84924 & 11744 & 40470 & 137138 \\
\hline & $\%$ & 22,08 & 11,74 & 19,58 & 20 \\
\hline \multirow{2}{*}{$600-900$} & ha & 101678 & 13550 & 48965 & 164193 \\
\hline & $\%$ & 26,44 & 13,54 & 23,69 & 24 \\
\hline \multirow{2}{*}{$900-1200$} & ha & 46975 & 1562 & 15671 & 64208 \\
\hline & $\%$ & 12,21 & 1,56 & 7,58 & 9 \\
\hline \multirow{2}{*}{$1200-1500$} & ha & 23469 & 162 & 20529 & 44159 \\
\hline & $\%$ & 6,10 & 0,16 & 9,93 & 6 \\
\hline \multirow{2}{*}{$1500-1800$} & ha & 15775 & 0 & 22305 & 38080 \\
\hline & $\%$ & 4,10 & 0,00 & 10,79 & 6 \\
\hline \multirow{2}{*}{$1800-2100$} & ha & 7885 & 0 & 22460 & 30345 \\
\hline & $\%$ & 2,05 & 0,00 & 10,87 & 4 \\
\hline \multirow{2}{*}{$2100-2400$} & ha & 2279 & 0 & 10223 & 12502 \\
\hline & $\%$ & 0,59 & 0,00 & 4,95 & 2 \\
\hline \multirow{2}{*}{$2400-2700$} & ha & 572 & 0 & 4197 & 4769 \\
\hline & $\%$ & 0,15 & 0,00 & 2,03 & 1 \\
\hline \multirow{2}{*}{$2700-3000$} & ha & 42 & 0 & 2915 & 2967 \\
\hline & $\%$ & 0,01 & 0,00 & 1,41 & 0 \\
\hline \multirow{2}{*}{$3000-3300$} & ha & 0 & 0 & 1400 & 1400 \\
\hline & $\%$ & 0,00 & 0,00 & 0,68 & 0 \\
\hline \multirow{2}{*}{$>3400$} & ha & 0 & 0 & 191 & 191 \\
\hline & $\%$ & 0,00 & 0,00 & 0,09 & 0 \\
\hline Together & ha & 384582 & 100047 & 206660 & 691289 \\
\hline
\end{tabular}


Table 2: The distribution of the study area (ha and \%) over the slopes of the terrain.

\begin{tabular}{|c|c|c|c|c|c|}
\hline \multirow{2}{*}{\multicolumn{2}{|c|}{ Height, m. н.у.м }} & \multirow{2}{*}{$\begin{array}{c}\text { Regions } \\
\text { Shemakha } \\
\end{array}$} & \multirow[b]{2}{*}{ Akhsuinsky } & \multirow[b]{2}{*}{ Ismailinskiy } & \multirow[t]{2}{*}{ Together } \\
\hline & & & & & \\
\hline \multirow{2}{*}{$0-6$} & ha & 187157 & 71131 & 61504 & 319792 \\
\hline & $\%$ & 48,67 & 71,10 & 29,76 & 46,26 \\
\hline \multirow{2}{*}{$6-10$} & ha & 88830 & 7393 & 28770 & 124993 \\
\hline & $\%$ & 23,10 & 7,39 & 13,92 & 18,08 \\
\hline \multirow{2}{*}{$10-18$} & ha & 87641 & 17033 & 53425 & 158099 \\
\hline & $\%$ & 22,79 & 17,02 & 25,85 & 22,87 \\
\hline \multirow{2}{*}{$18-27$} & ha & 17782 & 4326 & 34060 & 56168 \\
\hline & $\%$ & 4,62 & 4,32 & 16,48 & 8,13 \\
\hline \multirow{2}{*}{$27-35$} & ha & 2855 & 164 & 20176 & 23195 \\
\hline & $\%$ & 0,74 & 0,16 & 9,76 & 3,36 \\
\hline \multirow{2}{*}{$>35$} & ha & 317 & 0 & 8724 & 9041 \\
\hline & $\%$ & 0,08 & 0,00 & 4,22 & 1,31 \\
\hline \multicolumn{2}{|c|}{ Together } & 384582 & 100047 & 206660 & 691289 \\
\hline
\end{tabular}

\section{Classes of the Slope of the Terrain, Depending on the Altitude Intervals}

Table 3: The distribution of the territory of the Shemakha district according to the grades of the terrain according to the altitude intervals.

\begin{tabular}{|c|c|c|c|c|c|c|c|c|c|c|c|c|c|}
\hline \multirow{3}{*}{$\begin{array}{c}\text { Height, m. } \\
\text { above sea } \\
\text { level }\end{array}$} & \multicolumn{12}{|c|}{ slope classes\% } & \multirow{3}{*}{ sum } \\
\hline & \multicolumn{2}{|c|}{$0-6$} & \multicolumn{2}{|c|}{$6-10$} & \multicolumn{2}{|c|}{$10-18$} & \multicolumn{2}{|c|}{$18-27$} & \multicolumn{2}{|c|}{$27-37$} & \multicolumn{2}{|c|}{$37-52$} & \\
\hline & ha & $\%$ & ha & $\%$ & ha & $\%$ & ha & $\%$ & ha & $\%$ & ha & $\%$ & \\
\hline 1 & 2 & 3 & 4 & 5 & 6 & 7 & 8 & 9 & 10 & 11 & 12 & 13 & 14 \\
\hline$<0$ & 31245 & 8.12 & 0 & 0,00 & 0 & 0,00 & 0 & 0,00 & 0 & 0,00 & 0 & 0,00 & 31245 \\
\hline $0-100$ & 37036 & 9,63 & 714 & 0,19 & 251 & 0,07 & 27 & 0,01 & 2 & 0,00 & 0 & 0,00 & 38030 \\
\hline $100-200$ & 13223 & 3,44 & 2852 & 0,74 & 1957 & 0,51 & 372 & 0,10 & 19 & 0,00 & 0 & 0,00 & 18422 \\
\hline $200-300$ & 4661 & 1,21 & 4076 & 1,06 & 3730 & 0,97 & 752 & 0,20 & 67 & 0,02 & 0 & 0,00 & 13285 \\
\hline $300-400$ & 7157 & 1,86 & 5037 & 1,31 & 5548 & 1,44 & 1159 & 0,30 & 73 & 0,02 & 0 & 0,00 & 18978 \\
\hline $400-500$ & 15741 & 4,09 & 7078 & 1,84 & 6609 & 1,72 & 1302 & 0,34 & 70 & 0,02 & 0 & 0,00 & 30800 \\
\hline $500-600$ & 16189 & 4,21 & 8845 & 2,30 & 8644 & 2,25 & 1420 & 0,37 & 52 & 0,01 & 0 & 0,00 & 35150 \\
\hline $600-700$ & 17033 & 4,43 & 11665 & 3,03 & 9814 & 2,55 & 1245 & 0,32 & 41 & 0,01 & 1 & 0,00 & 39799 \\
\hline $700-800$ & 16708 & 4,34 & 12164 & 3,16 & 8375 & 2,18 & 890 & 0,23 & 24 & 0,01 & 1 & 0,00 & 38162 \\
\hline $800-900$ & 7497 & 1,95 & 8050 & 2,09 & 7152 & 1,86 & 962 & 0,25 & 55 & 0,01 & 1 & 0,00 & 23717 \\
\hline $900-1000$ & 4810 & 1,25 & 6280 & 1,63 & 5615 & 1,46 & 786 & 0,20 & 100 & 0,03 & 5 & 0,00 & 17596 \\
\hline $1000-1100$ & 4415 & 1,15 & 4804 & 1,25 & 5480 & 1,43 & 658 & 0,17 & 97 & 0,03 & 11 & 0,00 & 15466 \\
\hline $1100-1200$ & 4078 & 1,06 & 4265 & 1,11 & 4780 & 1,24 & 637 & 0,17 & 128 & 0,03 & 25 & 0,01 & 13914 \\
\hline $1200-1300$ & 3402 & 0,88 & 3522 & 0,92 & 3348 & 0,84 & 424 & 0,11 & 101 & 0,03 & 18 & 0,00 & 10816 \\
\hline $1300-1400$ & 1625 & 0,42 & 2665 & 0,69 & 2605 & 0,68 & 287 & 0,07 & 79 & 0,02 & 25 & 0,01 & 7286 \\
\hline $1400-1500$ & 722 & 0,19 & 1880 & 0,49 & 2342 & 0,61 & 342 & 0,09 & 73 & 0,02 & 9 & 0,00 & 5368 \\
\hline $1500-1600$ & 472 & 0,12 & 1765 & 0,46 & 2806 & 0,73 & 634 & 0,16 & 63 & 0,02 & 1 & 0,00 & 5741 \\
\hline $1600-1700$ & 497 & 0,13 & 1161 & 0,30 & 2885 & 0,75 & 833 & 0,22 & 58 & 0,02 & 2 & 0,00 & 5437 \\
\hline $1700-1800$ & 290 & 0,08 & 988 & 0,26 & 2183 & 0,57 & 1063 & 0,28 & 73 & 0,02 & 1 & 0,00 & 4597 \\
\hline
\end{tabular}




\begin{tabular}{|c|c|c|c|c|c|c|c|c|c|c|c|c|c|}
\hline $1800-1900$ & 230 & 0,06 & 650 & 0,17 & 1654 & 0,43 & 1040 & 0,27 & 133 & 0,03 & 1 & 0,00 & 3708 \\
\hline $1900-2000$ & 88 & 0,02 & 242 & 0,06 & 991 & 0,26 & 998 & 0,26 & 203 & 0,05 & 1 & 0,00 & 2523 \\
\hline $2000-2100$ & 32 & 0,01 & 69 & 0,02 & 439 & 0,11 & 741 & 0,19 & 357 & 0,09 & 17 & 0,00 & 1655 \\
\hline $2100-2200$ & 1 & 0,00 & 17 & 0,00 & 194 & 0,05 & 521 & 0,14 & 346 & 0,09 & 45 & 0,01 & 1124 \\
\hline $2200-2300$ & 2 & 0,00 & 16 & 0,00 & 102 & 0,03 & 353 & 0,09 & 264 & 0,07 & 46 & 0,01 & 782 \\
\hline $2300-2400$ & 5 & 0,00 & 4 & 0,00 & 28 & 0,01 & 132 & 0,03 & 156 & 0,04 & 49 & 0,01 & 373 \\
\hline 1 & 2 & 3 & 4 & 5 & 6 & 7 & 8 & 9 & 10 & 11 & 12 & 13 & 14 \\
\hline $2400-2500$ & 0 & 0,00 & 11 & 0,00 & 42 & 0,01 & 92 & 0,02 & 111 & 0,03 & 27 & 0,01 & 282 \\
\hline $2500-2600$ & 0 & 0,00 & 7 & 0,00 & 27 & 0,01 & 75 & 0,02 & 62 & 0,02 & 24 & 0,01 & 197 \\
\hline $2600-2700$ & 0 & 0,00 & 3 & 0,00 & 20 & 0,01 & 25 & 0,01 & 41 & 0,01 & 5 & 0,00 & 94 \\
\hline $2700-2800$ & 0 & 0,00 & 1 & 0,00 & 14 & 0,00 & 12 & 0,00 & 7 & 0,00 & 2 & 0,00 & 35 \\
\hline $2800-2900$ & 0 & 0,00 & 1 & 0,00 & 3 & 0,00 & 2 & 0,00 & 0 & 0,00 & 0 & 0,00 & 7 \\
\hline Sum & 187157 & 48,67 & 88830 & 23,10 & 87641 & 22,79 & 17782 & 4,62 & 2855 & 0,74 & 317 & 0,08 & 384582 \\
\hline
\end{tabular}

Table 4: Distribution of the territory of the Akhsu region according to the grade of the terrain depending on the altitude interval.

\begin{tabular}{|c|c|c|c|c|c|c|c|c|c|c|c|}
\hline \multirow{3}{*}{$\begin{array}{l}\text { Height, } \mathrm{m} \text {. } \\
\text { above sea level }\end{array}$} & \multicolumn{10}{|c|}{ Slope classes $\%$} & \multirow{3}{*}{ Sum } \\
\hline & \multicolumn{2}{|c|}{$0-6$} & \multicolumn{2}{|c|}{$6-10$} & \multicolumn{2}{|c|}{$10-18$} & \multicolumn{2}{|c|}{$18-27$} & \multicolumn{2}{|c|}{$27-35$} & \\
\hline & га & $\%$ & га & $\%$ & га & $\%$ & га & $\%$ & га & $\%$ & \\
\hline$<100$ & 52626 & 52,60 & 16 & 0,02 & 13 & 0,01 & 0 & 0,00 & 0 & 0,00 & 52656 \\
\hline $100-200$ & 12380 & 12,37 & 817 & 0,82 & 485 & 0,49 & 74 & 0,07 & 0 & 0,00 & 13757 \\
\hline $200-300$ & 3315 & 3,31 & 1150 & 1,15 & 1830 & 1,83 & 317 & 0,32 & 5 & 0,01 & 6617 \\
\hline $300-400$ & 418 & 0,42 & 693 & 0,69 & 2051 & 2,05 & 507 & 0,51 & 19 & 0,02 & 3687 \\
\hline $400-500$ & 215 & 0,21 & 390 & 0,39 & 2220 & 2,22 & 834 & 0,83 & 13 & 0,01 & 3672 \\
\hline $500-600$ & 273 & 0,27 & 538 & 0,54 & 2594 & 2,59 & 974 & 0,97 & 5 & 0,01 & 4384 \\
\hline $600-700$ & 327 & 0,33 & 575 & 0,76 & 3338 & 3,34 & 846 & 0,88 & 20 & 0,02 & 5318 \\
\hline $700-800$ & 690 & 0,69 & 1516 & 1,52 & 2689 & 2,69 & 406 & 0,41 & 22 & 0,02 & 5324 \\
\hline $800-900$ & 681 & 0,68 & 988 & 0,99 & 1060 & 1,06 & 166 & 0,17 & 13 & 0,01 & 2909 \\
\hline $900-1000$ & 154 & 0,15 & 254 & 0,28 & 241 & 0,24 & 79 & 0,08 & 16 & 0,02 & 775 \\
\hline $1000-1100$ & 29 & 0,03 & 198 & 0,20 & 281 & 0,28 & 27 & 0,03 & 16 & 0,02 & 551 \\
\hline $1100-1200$ & 23 & 0,02 & 36 & 0,04 & 127 & 0,13 & 32 & 0,03 & 17 & 0,02 & 236 \\
\hline $1200-1300$ & 0 & 0,00 & 7 & 0,01 & 82 & 0,08 & 29 & 0,03 & 13 & 0,01 & 131 \\
\hline $1300-1400$ & 0 & 0,00 & 4 & 0,00 & 20 & 0,02 & 5 & 0,00 & 3 & 0,00 & 31 \\
\hline Sum & 71131 & 71,1 & 7393 & 7,39 & 17033 & 17,02 & 4326 & 4,32 & 164 & 0,16 & 100047 \\
\hline
\end{tabular}

Table 5: The distribution of the territory of the Isma yilli region according to the grades of the terrain according to the altitude intervals.

\begin{tabular}{|c|c|c|c|c|c|c|c|c|c|c|c|c|c|}
\hline \multirow{3}{*}{$\begin{array}{c}\text { Height, m. } \\
\text { above sea } \\
\text { level }\end{array}$} & \multicolumn{12}{|c|}{ Slope classes $\%$} & \multirow{3}{*}{ Sum } \\
\hline & \multicolumn{2}{|c|}{$<6$} & \multicolumn{2}{|c|}{$6-10$} & \multicolumn{2}{|c|}{$10-18$} & \multicolumn{2}{|c|}{$18-27$} & \multicolumn{2}{|c|}{$27-37$} & \multicolumn{2}{|c|}{$37-68$} & \\
\hline & Га & $\%$ & га & $\%$ & га & $\%$ & га & $\%$ & га & $\%$ & га & $\%$ & \\
\hline 1 & 2 & 3 & 4 & 5 & 6 & 7 & 8 & 9 & 10 & 11 & 12 & 13 & 14 \\
\hline$<100$ & 1849 & 0,89 & 321 & 0,16 & 92 & 04 & 0 & 0.00 & 0 & 0,00 & 0 & 0,00 & 2262 \\
\hline $100-200$ & 624 & 0,30 & 376 & 0,18 & 484 & 0.23 & 51 & 0.02 & 10 & 0.01 & 0 & 0,00 & 1546 \\
\hline $200-300$ & 10302 & 4,98 & 1937 & 0,94 & 1065 & 0.52 & 194 & 0.09 & 29 & 0.01 & 0 & 0,00 & 13,527 \\
\hline $300-400$ & 3303 & 1,60 & 2322 & 1,12 & 2564 & 1.24 & 288 & 0.14 & 26 & 0,01 & 3 & 0,00 & 8507 \\
\hline 400-500 & 4112 & 1,99 & 2019 & 0,98 & 3240 & 1.57 & 569 & 0.29 & 30 & 0,01 & 2 & 0,00 & 9999 \\
\hline $500-600$ & 13759 & 6,66 & 28668 & 1,39 & 4220 & $2 . .04$ & 1090 & 0.53 & 26 & 0,01 & 1 & 0,00 & 21964 \\
\hline $600-700$ & 13807 & 6,68 & 4012 & 1,94 & 4640 & 2.25 & 982 & 0.48 & 32 & 0,02 & 3 & 0,00 & 23476 \\
\hline $700-800$ & 8587 & 4,15 & 4068 & 1,97 & 3569 & 1.73 & 841 & 0.41 & 82 & 0,04 & 7 & 0,00 & 17154 \\
\hline $800-900$ & 2114 & 1,02 & 2386 & 1,15 & 2679 & 1.30 & 985 & 0.48 & 147 & 0,07 & 25 & 0,01 & 8336 \\
\hline
\end{tabular}




\begin{tabular}{|c|c|c|c|c|c|c|c|c|c|c|c|c|c|}
\hline $900-1000$ & 872 & 0,42 & 1190 & 0,58 & 2029 & 0.98 & 1130 & 0.55 & 349 & 0,17 & 48 & 0,02 & 5617 \\
\hline $1000-1100$ & 414 & 0,20 & 740 & 0,36 & 1699 & 0.82 & 1446 & 0.70 & 492 & 0,24 & 96 & 0,05 & 4887 \\
\hline $1100-1200$ & 194 & 0,09 & 552 & 0,27 & 1713 & 0.83 & 1776 & 0.86 & 776 & 0,38 & 158 & 0,08 & 5167 \\
\hline $1200-1300$ & 212 & 0,10 & 621 & 0,30 & 2015 & 0.98 & 2117 & 1.02 & 1025 & 0,50 & 191 & 0,09 & 6181 \\
\hline $1300-1400$ & 182 & 0,09 & 720 & 0,35 & 2553 & 1.24 & 2269 & 1.10 & 1154 & 0,56 & 237 & 0,11 & 7114 \\
\hline $1400-1500$ & 177 & 0,09 & 695 & 0,32 & 2440 & 1.18 & 2325 & 1.13 & 1359 & 0,66 & 276 & 0,13 & 7233 \\
\hline $1500-1600$ & 119 & 0,6 & 547 & 0,26 & 2499 & 1.21 & 2275 & 1.10 & 1429 & 0,69 & 376 & 0,18 & 7244 \\
\hline $1600-1700$ & 152 & 0,7 & 558 & 0,27 & 2440 & 1.18 & 2394 & 1.16 & 1527 & 0,74 & 455 & 0,22 & 7527 \\
\hline $1700-1800$ & 154 & 0,07 & 520 & 0,25 & 2490 & 1.21 & 2339 & 1.12 & 1561 & 0,76 & 448 & 0,24 & 7534 \\
\hline $1800-1900$ & 153 & 0,07 & 610 & 0,30 & 2743 & 1.32 & 2440 & 1.18 & 1522 & 0,74 & 533 & 0,26 & 7991 \\
\hline $1900-2000$ & 174 & 0,08 & 638 & 0,31 & 2682 & 1.30 & 2112 & 1.18 & 1268 & 0,61 & 604 & 0,29 & 7806 \\
\hline $2000-2100$ & 86 & 0,04 & 405 & 0,20 & 2355 & 1.14 & 1439 & 1.02 & 1130 & 0,55 & 573 & 0,28 & 6662 \\
\hline $2100-2200$ & 81 & 0,04 & 359 & 0,17 & 1723 & 0.83 & 661 & 0.70 & 1057 & 0,51 & 572 & 0,28 & 5231 \\
\hline $2200-2300$ & 59 & 0,03 & 246 & 0,12 & 769 & 0.37 & 2439 & 0.32 & 794 & 0,38 & 619 & 0,30 & 3148 \\
\hline $2300-2400$ & 7 & 0,00 & 30 & 0,01 & 156 & 0.08 & 373 & 0.18 & 682 & 0.33 & 596 & 0.29 & 1844 \\
\hline $2400-2500$ & 2 & 0,00 & 9 & 0,00 & 53 & 0.03 & 217 & 0.10 & 674 & 0.33 & 569 & 0.28 & 1523 \\
\hline 1 & 20 & 3 & 4 & 5 & 6 & 7 & 8 & 9 & 10 & 11 & 12 & 13 & 14 \\
\hline $2500-2600$ & 0 & 0,00 & 2 & 0,00 & 52 & 0,02 & 232 & 0,11 & 603 & 0,29 & 562 & 0,27 & 1451 \\
\hline $2600-2700$ & 2 & 0,00 & 6 & 0,00 & 42 & 0,02 & 172 & 0,08 & 557 & 0,27 & 446 & 0,22 & 1223 \\
\hline $2700-2800$ & 1 & 0,00 & 5 & 0,00 & 65 & 0,03 & 168 & 0,08 & 509 & 0,25 & 394 & 0,19 & 1141 \\
\hline $2800-2900$ & 0 & 0,00 & 10 & 0,00 & 66 & 0,03 & 141 & 0,07 & 382 & 0,18 & 317 & 0,15 & 916 \\
\hline $2900-3000$ & 6 & 0,00 & 7 & 0,00 & 77 & 0,04 & 173 & 0,08 & 329 & 0,16 & 267 & 0,13 & 858 \\
\hline $3000-3100$ & 1 & 0,00 & 21 & 0,00 & 84 & 0,04 & 183 & 0,09 & 292 & 0,14 & 174 & 0,08 & 754 \\
\hline $3100-3200$ & 2 & 0,00 & 2 & 0,00 & 59 & 0,03 & 110 & 0,05 & 177 & 0,09 & 98 & 0,05 & 448 \\
\hline $3200-3300$ & 0 & 0,00 & 3 & 0,00 & 34 & 0,02 & 59 & 0,03 & 76 & 0,04 & 26 & 0,01 & 198 \\
\hline $3300-3400$ & 0 & 0,00 & 3 & 0,00 & 35 & 0,02 & 38 & 0,02 & 42 & 0,02 & 4 & 0,00 & 122 \\
\hline $3400-3500$ & 0 & 0,00 & 0 & 0,00 & 3 & 0,00 & 16 & 0,01 & 21 & 0,01 & 2 & 0,00 & 42 \\
\hline $3500-3600$ & 0 & 0,00 & 2 & 0,00 & 4 & 0,00 & 11 & 0,01 & 11 & 0,01 & 0 & 0,00 & 28 \\
\hline Sum & 61504 & 29,76 & 28,770 & 13,92 & 53425 & 25,85 & 34060 & 16,48 & 20176 & 9,76 & 8724 & 4,22 & 206660 \\
\hline
\end{tabular}

Tables 3-5 contain data that allow analyzing the distribution of the slope of the terrain along altitude intervals. This distribution was prepared for all administrative units of the area under consideration. In the Shemakha region, a clear dependence is shown, an increase in the slope, along with an increase in altitude above sea level. Up to a height of $1200 \mathrm{~m}$ there are lands with a slight slope-up to $6 \%$ (3.50). They occupy $49 \%$ of the area. Territories here with a slope of $6-10 \%$ and $10-18 \%$ are located at an altitude of $400-1700$ m. M.u.m. about $23 \%$ of the area's area. Areas with a slope of more than $18 \%$ make up more than $5 \%$ of the total area and are located in the highlands. In the Aksuinsky area, the terrain with an insignificant slope of up to $6 \%$ (3.50) prevails and they are located mainly at an altitude of $300 \mathrm{~m}$ above sea level - this is $71 \%$ of the total area. Areas with a slope of more than $6 \%$ are located at altitudes from 300 to $1400 \mathrm{~m}$ above sea level - about 29\%. Clearly marked (17\% of the total area) of land with a slope of $10-18 \%$ (6100) (Table 4).

In the Ismayilli region, most of the territory with a slope of $<6$ to $10 \%$ is located at an altitude of 300 to $900 \mathrm{~m}$ above sea level.
Areas with a slope of 10 to $37 \%$ occur at an altitude of 3000 meters above sea level. Locations with a slope of more than $37 \% \%$ are located at the highest altitude but make up only $4 \%$ of the total area of the area. The second, from the thematic layers created in the database, is the land use of the study area. Due to the availability of cartographic materials, it was considered exclusively for the territory of the Akhsu district.

Analysis of the structure of land use in the Akhsu district showed that it is purely agricultural. The area is dominated by arable land, which makes up about 50\% and tilled areas, which include pastures, meadows and degrees - more than $23 \%$ of the total area. Agricultural lands are supplemented with vegetable gardens, orchards and vineyards, occupying 3\% of the area. There are few forests and shrubs, in general, about $12 \%$. There are also areas completely devoid of vegetation in the Akhsu district, about $4 \%$ of them. A small part is occupied by water reservoirs, wetlands and urban and rural areas, amounting to $6.9 \%$ of the total area (Tables 6-8) provide information on the distribution of land use patterns over altitudinal intervals and grades of terrain slopes. 
Most agricultural land is located at a height of up to $400 \mathrm{~m}$ above sea level. Only pastures are located up to an altitude of 1400 meters above sea level. forest territories are in all altitude intervals. The remaining forms of land use are located at lower altitudes (Table

Table 6: Land use in Ahsuinsky district.
7). Considering the slope of the slopes, most of the territories are in the gradient class $0-6 \%$ - almost $71 \%$. Only pastures, green lands, as well as deciduous forests, are located on slopes with a higher slope (Table 8).

\begin{tabular}{|c|c|c|}
\hline Land use & ha & $\%$ \\
\hline The Cities & 1237 & 1,24 \\
\hline Pshchenitsa & 49979 & 49,96 \\
\hline Gardens & 1123 & 1,12 \\
\hline Vineyards & 528 & 0,53 \\
\hline Arable land & 52868 & 52,84 \\
\hline Pastures & 12394 & 12,39 \\
\hline Green grounds & 6511 & 6,51 \\
\hline Power & 4708 & 4,71 \\
\hline Dry dried valleys & 673 & 0,67 \\
\hline Coniferous forests & 535 & 0,53 \\
\hline Deciduous forests & 8451 & 8,45 \\
\hline Mixed forests & 216 & 0,22 \\
\hline Marshes & 844 & 0,84 \\
\hline Reservoirs & 27 & 0,03 \\
\hline Stavy & 25 & 0,02 \\
\hline Piece of lake & 80 & 0,08 \\
\hline Bays & 1137 & 1,14 \\
\hline Shrubs & 2668 & 2,67 \\
\hline Soil without vegetation & 248 & 0,25 \\
\hline Rocks & 3827 & 3,38 \\
\hline Rural low construction & 527 & 0,53 \\
\hline City low building & 3919 & 3,92 \\
\hline High Urban Construction & 147 & 0,15 \\
\hline Rockstones, mines & 241 & 0,24 \\
\hline Together & 100047 & 10000 \\
\hline
\end{tabular}

Table 7: Land use by altitude intervals.

\begin{tabular}{|c|c|c|c|c|c|c|c|c|c|c|c|c|c|c|c|c|}
\hline \multirow{3}{*}{ Land use } & \multicolumn{16}{|c|}{ Height, $\mathbf{m}$. above sea level } \\
\hline & \multicolumn{2}{|c|}{$<100$} & \multicolumn{2}{|c|}{$100-200$} & \multicolumn{2}{|c|}{$200-300$} & \multicolumn{2}{|c|}{$300-400$} & \multicolumn{2}{|c|}{$400-500$} & \multicolumn{2}{|c|}{$500-600$} & \multicolumn{2}{|c|}{$600-700$} & \multicolumn{2}{|c|}{$700-800$} \\
\hline & ha & $\%$ & ha & $\%$ & ha & $\%$ & ha & $\%$ & ha & $\%$ & ha & $\%$ & ha & $\%$ & ha & $\%$ \\
\hline 1 & 2 & 3 & 4 & 5 & 6 & 7 & 8 & 9 & 10 & 11 & 12 & 13 & 14 & 15 & 16 & 17 \\
\hline The Cities & 231 & 0,23 & 953 & 0,95 & 53 & 0,05 & 0 & 0,00 & 0 & 0,00 & 0 & 0,00 & 0 & 0,00 & 0 & 0,00 \\
\hline Pshchenitsa & 42825 & 42,81 & 5607 & 5,60 & 690 & 0,69 & 235 & 0,23 & 0 & 0,00 & 0 & 0,00 & 7 & 0,01 & 142 & 0,14 \\
\hline Gardens & 339 & 0,34 & 701 & 0,70 & 42 & 0,04 & 0 & 0,00 & 0 & 0,00 & 0 & 0,00 & 1 & 0,00 & 41 & 0,04 \\
\hline Vineyards & 19 & 0,02 & 113 & 0,11 & 306 & 0,31 & 40 & 0,04 & 11 & 0,01 & 0 & 0,00 & 0 & 0,00 & 35 & 0,04 \\
\hline Pastures & 438 & 0,44 & 1635 & 1,63 & 461 & 0,46 & 511 & 0,51 & 691 & 0,69 & 1392 & 1,39 & 2385 & 2,38 & 2623 & 2,62 \\
\hline Green grounds & 42 & 0,04 & 596 & 0,60 & 1345 & 1,34 & 1418 & 1,42 & 1239 & 1,24 & 873 & 0,87 & 495 & 0,49 & 31 & 0,31 \\
\hline Power & 3188 & 3,19 & 1459 & 1,46 & 61 & 0,06 & 0 & 0,00 & 0 & 0,00 & 0 & 0,00 & 0 & 0,00 & 0 & 0,00 \\
\hline $\begin{array}{l}\text { Dry Dried Loose } \\
\text { Valleys }\end{array}$ & 223 & 0,22 & 242 & 0,24 & 201 & 0,20 & 6 & 0,01 & 0 & 0,00 & 0 & 0,00 & 0 & 0,00 & 0 & 0,00 \\
\hline Coniferous forests & 535 & 0,53 & 0 & 0,00 & 0 & 0,00 & 0 & 0,00 & 0 & 0,00 & 0 & 0,00 & 0 & 0,00 & 0 & 0,00 \\
\hline Deciduous forests & 0 & 0,00 & 93 & 0,09 & 62 & 0,06 & 421 & 0,42 & 1018 & 1,02 & 1546 & 1,55 & 1928 & 1,93 & 1901 & 1,90 \\
\hline Mixed forests & 33 & 0,03 & 76 &, 08 & 104 & 0,10 & 0 & 0,00 & 0 & 0,00 & 3 & 0,00 & 0 & 0,00 & 0 & 0,00 \\
\hline
\end{tabular}




\begin{tabular}{|c|c|c|c|c|c|c|c|c|c|c|c|c|c|c|c|c|}
\hline Marshes & 844 & 0,84 & 0 & 0,00 & 0 & 0,00 & 0 & 0,00 & 0 & 0,00 & 0 & 0,00 & 0 & 0,00 & 0 & 0,00 \\
\hline Reservoirs & 0 & 0,00 & 27 & 0,03 & 0 & 0,00 & 0 & 0,00 & 0 & 0,00 & 0 & 0,00 & 0 & 0,00 & 0 & 0,00 \\
\hline Stavy & 23 & 0,02 & 0 & 0,00 & 0 & 0,00 & 0 & 0,00 & 0 & 0,00 & 0 & 0,00 & 0 & 0,00 & 2 & 0,00 \\
\hline Piece of lake & 0 & 0,00 & 80 & 0,08 & 0 & 0,00 & 0 & 0,00 & 0 & 0,00 & 0 & 0,00 & 0 & 0,00 & 0 & 0,00 \\
\hline Bays & 0 & 0,00 & 82 & 0,08 & 289 & 0,29 & 165 & 0,16 & 96 & 0,10 & 196 & 0,20 & 230 & 0,23 & 78 & 0,08 \\
\hline Shrubs & 1110 & 1,11 & 312 & 0,31 & 1244 & 1,24 & 2 & 0,00 & 0 & 0,00 & 0 & 0,00 & 0 & 0,00 & 0 & 0,00 \\
\hline $\begin{array}{c}\text { The soil layer } \\
\text { without vegetation }\end{array}$ & 138 & 0,14 & 56 & 0,06 & 53 & 0,05 & 1 & 0,00 & 0 & 0,00 & 0 & 0,00 & 0 & 0,00 & 0 & 0,00 \\
\hline Rocks & 1 & 0,00 & 414 & 0,41 & 1114 & 1,11 & 904 & 0,90 & 627 & 0,63 & 372 & 0,37 & 273 & 0,24 & 136 & 0,14 \\
\hline $\begin{array}{l}\text { Rural low } \\
\text { construction }\end{array}$ & 0 & 0,00 & 219 & 0,22 & 45 & 0,04 & 8 & 0,01 & 0 & 0,00 & 0 & 0,00 & 16 & 0,02 & 38 & 0,04 \\
\hline City low building & 2383 & 2,38 & 905 & 0,90 & 541 & 0,54 & 2 & 0,00 & 12 & 0,01 & 25 & 0,03 & 23 & 0,02 & 28 & 0,03 \\
\hline $\begin{array}{l}\text { High Urban } \\
\text { Construction }\end{array}$ & 147 & 0,15 & 0 & 0,00 & 0 & 0,00 & 0 & 0,00 & 0 & 0,00 & 0 & 0,00 & 0 & 0,00 & 0 & 0,00 \\
\hline Rockstones, mines & 0 & 0,00 & 217 & 0,22 & 24 & 0,02 & 0 & 0,00 & 0 & 0,00 & 0 & 0,00 & 0 & 0,00 & 0 & 0,00 \\
\hline Together & 52521 & 52,50 & 13786 & 13,78 & 6636 & 6,60 & 3713 & 3,71 & 3696 & 3,69 & 4408 & 4,41 & 5322 & 5,32 & 5333 & 5,33 \\
\hline \multirow{3}{*}{ Land use } & \multicolumn{12}{|c|}{ Height, $\mathrm{m}$. above sea level } & \multirow{2}{*}{\multicolumn{2}{|c|}{ Вместе }} & & \\
\hline & \multicolumn{2}{|c|}{$800-900$} & \multicolumn{2}{|c|}{$900-1000$} & \multicolumn{2}{|c|}{$1000-1100$} & \multicolumn{2}{|c|}{$1100-1200$} & \multicolumn{2}{|c|}{$1200-1300$} & \multicolumn{2}{|c|}{$1300-1400$} & & & & \\
\hline & ha & $\%$ & ha & $\%$ & ha & $\%$ & ha & $\%$ & ha & $\%$ & ha & $\%$ & ha & $\%$ & & \\
\hline 1 & 2 & 3 & 4 & 5 & 6 & 7 & 8 & 9 & 10 & 11 & 12 & 13 & 16 & 17 & & \\
\hline The Cities & 0 & 0,00 & 0 & 0,00 & 0 & 0,00 & 0 & 0,00 & 0 & 0,00 & 0 & 0,00 & 1237 & 1,24 & & \\
\hline Pshchenitsa & 367 & 0,37 & 105 & 0,11 & 1 & 0,00 & 0 & 0,00 & 0 & 0,00 & 0 & 0,00 & 49979 & 49,96 & & \\
\hline Gardens & 0 & 0,00 & 0 & 0,00 & 0 & 0,00 & 0 & 0,00 & 0 & 0,00 & 0 & 0,00 & 1123 & 1,12 & & \\
\hline Vineyards & 4 & 0,00 & 0 & 0,00 & 0 & 0,00 & 0 & 0,00 & 0 & 0,00 & 0 & 0,00 & 528 & 0,53 & & \\
\hline Pastures & 1530 & 1,53 & 384 & 0,38 & 217 & 0,22 & 98 & 0,10 & 28 & 0,03 & 1 & 0,00 & 12394 & 12,39 & & \\
\hline Green grounds & 189 & 0,19 & 3 & 0,00 & 0 & 0,00 & 0 & 0,00 & 0 & 0,00 & 0 & 0,00 & 6511 & 6,51 & & \\
\hline Power & 0 & 0,00 & 0 & 0,00 & 0 & 0,00 & 0 & 0,00 & 0 & 0,00 & 0 & 0,00 & 4708 & 4,71 & & \\
\hline $\begin{array}{l}\text { Dry Dried Loose } \\
\text { Valleys }\end{array}$ & 0 & 0,00 & 0 & 0,00 & 0 & 0,00 & 0 & 0,00 & 0 & 0,00 & 0 & 0,00 & 673 & 0,67 & & \\
\hline Coniferous forests & 0 & 0,00 & 0 & 0,00 & 0 & 0,00 & 0 & 0,00 & 0 & 0,00 & 0 & 0,00 & 535 & 0,53 & & \\
\hline Deciduous forests & 617 & 0,62 & 260 & 0,26 & 334 & 0,33 & 137 & 0,14 & 103 & 0,10 & 30 & 0,03 & 8451 & 8,45 & & \\
\hline Mixed forests & 0 & 0,00 & 0 & 0,00 & 0 & 0,00 & 0 & 0,00 & 0 & 0,00 & 0 & 0,00 & 216 & 0,22 & & \\
\hline Marshes & 0 & 0,00 & 0 & 0,00 & 0 & 0,00 & 0 & 0,00 & 0 & 0,00 & 0 & 0,00 & 844 & 0,84 & & \\
\hline Reservoirs & 0 & 0,00 & 0 & 0,00 & 0 & 0,00 & 0 & 0,00 & 0 & 0,00 & 0 & 0,00 & 27 & 0,03 & & \\
\hline Stavy & 0 & 0,00 & 0 & 0,00 & 0 & 0,00 & 0 & 0,00 & 0 & 0,00 & 0 & 0,00 & 25 & 0,02 & & \\
\hline Piece of lake & 0 & 0,00 & 0 & 0,00 & 0 & 0,00 & 0 & 0,00 & 0 & 0,00 & 0 & 0,00 & 80 & 0,08 & & \\
\hline Bays & 0 & 0,00 & 0 & 0,00 & 0 & 0,00 & 0 & 0,00 & 0 & 0,00 & 0 & 0,00 & 1137 & 1,14 & & \\
\hline Shrubs & 0 & 0,00 & 0 & 0,00 & 0 & 0,00 & 0 & 0,00 & 0 & 0,00 & 0 & 0,00 & 2668 & 2,67 & & \\
\hline $\begin{array}{c}\text { The soil layer } \\
\text { without vegetation }\end{array}$ & 0 & 0,00 & 0 & 0,00 & 0 & 0,00 & 0 & 0,00 & 0 & 0,00 & 0 & 0,00 & 248 & 0,25 & & \\
\hline Rocks & 21 & 0,02 & 0 & 0,00 & 0 & 0,00 & 0 & 0,00 & 0 & 0,00 & 0 & 0,00 & 3827 & 3,83 & & \\
\hline $\begin{array}{c}\text { Rural low } \\
\text { construction }\end{array}$ & 180 & 0,18 & 22 & 0,02 & 0 & 0,00 & 0 & 0,00 & 0 & 0,00 & 0 & 0,00 & 527 & 0,53 & & \\
\hline City low building & 0 & 0,00 & 0 & 0,00 & 0 & 0,00 & 0 & 0,00 & 0 & 0,00 & 0 & 0,00 & 3919 & 3,92 & & \\
\hline $\begin{array}{l}\text { High Urban } \\
\text { Construction }\end{array}$ & 0 & 0,00 & 0 & 0,00 & 0 & 0,00 & 0 & 0,00 & 0 & 0,00 & 0 & 0,00 & 147 & 0,15 & & \\
\hline Rockstones, mines & 0 & 0,00 & 0 & 0,00 & 0 & 0,00 & 0 & 0,00 & 0 & 0,00 & 0 & 0,00 & 241 & 0,24 & & \\
\hline Together & 2909 & 2,91 & 775 & 0,77 & 551 & 0,55 & 236 & 0,24 & 131 & 0,13 & 31 & 0,03 & 100047 & 100,0 & & \\
\hline
\end{tabular}


Table 8: Land use according to grades of the slope of the terrain.

\begin{tabular}{|c|c|c|c|c|c|c|c|c|c|c|c|c|}
\hline \multirow{3}{*}{ Land use } & \multicolumn{10}{|c|}{ Classes slopes\% } & \multirow{2}{*}{\multicolumn{2}{|c|}{ Together }} \\
\hline & \multicolumn{2}{|c|}{$0-6$} & \multicolumn{2}{|c|}{$6-10$} & \multicolumn{2}{|c|}{$10-18$} & \multicolumn{2}{|c|}{$18-27$} & \multicolumn{2}{|c|}{$27-37$} & & \\
\hline & ha & $\%$ & ha & $\%$ & ha & $\%$ & ha & $\%$ & ha & $\%$ & ha & $\%$ \\
\hline The Cities & 49085 & 49,06 & 533,2 & 0,53 & 348 & 0,35 & 13 & 0,01 & 1 & 0,00 & 49979 & 49,96 \\
\hline Pshchenitsa & 1113 & 1,11 & 105,3 & 0,11 & 19 & 0,02 & 0 & 0,00 & 0 & 0,00 & 1237 & 1,24 \\
\hline Gardens & 1083 & 1,08 & 32,8 & 0,03 & 8 & 0,01 & 0 & 0,00 & 0 & 0,00 & 1123 & 1,12 \\
\hline Vineyards & 371 & 0,37 & 51,2 & 0,05 & 90 & 0,09 & 16 & 0,02 & 0 & 0,00 & 528 & 0,53 \\
\hline Pastures & 2895 & 2,89 & 2602,5 & 2,60 & 5956 & 5,95 & 932 & 0,93 & 8 & 0,01 & 12394 & 12,39 \\
\hline Green grounds & 658 & 0,66 & 1462,2 & 1,46 & 3486 & 3,48 & 893 & 0,89 & 12 & 0,01 & 6511 & 6,51 \\
\hline Power & 4632 & 4,63 & 53,8 & 0,05 & 19 & 0,02 & 3 & 0,00 & 0 & 0,00 & 4708 & 4,71 \\
\hline Dry Dried Loose Valleys & 642 & 0,64 & 5,8 & 0,01 & 12 & 0,01 & 12 & 0,01 & 0 & 0,00 & 673 & 0,67 \\
\hline Coniferous forests & 535 & 0,53 & 0,0 & 0,00 & 0 & 0,00 & 0 & 0,00 & 0 & 0,00 & 535 & 0,53 \\
\hline Deciduous forests & 595 & 0,60 & 1565,0 & 1,56 & 4655 & 4,65 & 1501 & 1,50 & 134 & 0,13 & 8451 & 8,45 \\
\hline Mixed forests & 208 & 0,21 & 1,7 & 0,00 & 6 & 0,01 & 11 & 0,00 & 0 & 0,00 & 216 & 0,22 \\
\hline Marshes & 844 & 0,84 & 0,0 & 0,00 & 0 & 0,00 & 0 & 0,00 & 0 & 0,00 & 844 & 0,84 \\
\hline Reservoirs & 27 & 0,03 & 0,0 & 0,00 & 0 & 0,00 & 0 & 0,00 & 0 & 0,00 & 27 & 0,03 \\
\hline Stavy & 25 & 0,02 & 0,4 & 0,00 & 0 & 0,00 & 0 & 0,00 & 0 & 0,00 & 25 & 0,02 \\
\hline Piece of lake & 78 & 0,08 & 1,9 & 0,00 & 0 & 0,00 & 0 & 0,00 & 0 & 0,00 & 80 & 0,08 \\
\hline Bays & 735 & 0,74 & 146,5 & 0,15 & 179 & 0,18 & 74 & 0,07 & 2 & 0,00 & 1137 & 1,14 \\
\hline Shrubs & 2550 & 2,55 & 25,3 & 0,03 & 62 & 0,06 & 31 & 0,03 & 0 & 0,00 & 2668 & 2,67 \\
\hline $\begin{array}{c}\text { The soil layer without } \\
\text { vegetation }\end{array}$ & 196 & 0,20 & 16,8 & 0,02 & 24 & 0,02 & 11 & 0,01 & 0 & 0,00 & 248 & 0,25 \\
\hline Rocks & 310 & 0,31 & 585,5 & 0,59 & 2051 & 2,05 & 872 & 0,87 & 8 & 0,01 & 3827 & 3,83 \\
\hline Rural low construction & 354 & 0,35 & 117,1 & 0,12 & 55 & 0,05 & 1 & 0,00 & 0 & 0,00 & 527 & 0,53 \\
\hline City low building & 3723 & 3,72 & 87,9 & 0,09 & 98 & 0,10 & 1 &, 01 & 0 & 0,00 & 3919 & 3,92 \\
\hline High Urban Construction & 147 & 0,15 & 0,0 & 0,00 & 0 & 0,00 & 0 & 0,00 & 0 & 0,00 & 147 & 0,15 \\
\hline Rockstones, mines & 217 & 0,22 & 8,1 & 0,01 & 16 & 0,02 & 0 & 0,00 & 0 & 0,00 & 241 & 0,24 \\
\hline Together & 71026 & 70,99 & 7403,2 & 7,40 & 17084 & 17,08 & 4369 & 4,37 & 165 & 0,17 & 100047 & 100,00 \\
\hline
\end{tabular}

\section{References}

1. Alekperov KA (1980) Soil-erosion map for the protection of lands. Moscow.

2. Aliev GA The problem of desertification in Azerbaijan, ways of solving it, Baku "Zia-Nurlan".

3. Aliev GA (1959) Soil of Azerbaijan. Publishing house Volobuev VR, AN Azerb. The USSR, pp. 122.

4. Aliev BG, Aliev ZG, Aliev IN (2000) Problems of erosion in Azerbaijan and ways to solve it. Baku: ZIMA-CPI “Nurlan”, pp. 122.

5. Armand DL (1956) Anthropogenic erosion processes. In the book. Agricultural erosion and the fight against it ed. An USSR, Moscow.

6. Babaeva KM(1995) Influence of simple and complex mineral fertilizers and lucerne sowing on restoration of fertility of eroded soils of the south-eastern slope of the Greater Caucasus, Dis Baku.

7. Bennet HH (1958) Fundamentals of soil protection.

8. Grossgeim AA (1948) Vegetative cover of the Caucasus. Publishing house of the Moscow Society of Naturalists, Moscow.

9. Huseynov AA (1991) Efficiency of surface improvement of eroded pastures. All-Union Scientific Conference, Dushanbe.
10. MN Zaslavsky (1969) To the draft classification of soils according to the degrees of erosion. Sat "Materials on the methodology of soil-erosion mapping of erosion control measures" Moscow.

11. M Zaslavsky (1983) Erosiology "high school".

12. Ibragimov AA Mapping of eroded soils on agricultural lands. Dashkesan district, Azerb, USSR.

13. Ibragimov AA (2000) Agroecological feature of eroded soils in Azerbaijan.

14. Materials on the study of erosion and irrigation and soil conservation in Azerbaijan, Baku.

15. Ibragimov AA (1972) Mapping of eroded soils on agricultural lands.

16. Groom G, Mücher CA, Margareta I, Thomas W (2006) Remote sensing inlandcape ecology: experience and perspectives in the European context. Landscape Ecology 21(3): 391-408.

17. Harris A, Carr AS, Dash J (2014) Remote sensing of vegetative integumentary dynamics and stability in southern Africa. International Journal of Earth Observation and Geoinformation 28: 131-139.

18. Klemas V (2013) The use of remote sensing for the selection and monitoring of wetland restoration sites: an overview. Journal of Coastal Research 29(4): 958-970. 


\section{(c) \\ This work is licensed under Creative Commons Attribution 4.0 License}

To Submit Your Article Click Here: Submit Article

DOI: 10.32474/OAJESS.2018.01.000102

$\begin{gathered}\text { Open Access Journal of Environmental } \\ \text { and Soil Sciences }\end{gathered}$
Assets of Publishing with us
- Global archiving of articles
- Immediate, unrestricted online access
- Rigorous Peer Review Process
- Authors Retain Copyrights
- Unique DOI for all articles

